\title{
On Deuling's Transition in a Nematic Liquid Crystal*
}

\author{
G. Barbero ${ }^{1}$ and A. Strigazzi ${ }^{2}$ \\ Dipartimento di Fisica, Politecnico, Torino, Italia \\ Z. Naturforsch. 39a, 571-574 (1984); received March 8, 1984
}

We analyse Deuling's problem both in the one constant approximation and in the general case. An explicit expression is found for the electric field threshold vs. the crossed applied magnetic field, in the strong anchoring hypothesis.

\section{Introduction}

We analyse the behaviour of a Nematic Liquid Crystal (NLC) with positive dielectric anisotropy, put in a planar cell subjected to crossed electric and magnetic field. In the hypothesis that the electric field $\boldsymbol{E}$ is normal to the bounding walls and the magnetic one, $\boldsymbol{H}$, is parallel to them and perpendicular to the initial molecular orientation (see Fig. 1), it is well known [ $1-3]$ that the arising elastic distortion presents a double threshold, i.e.:

1) If $H>H_{\mathrm{c}}$, in the NLC film a twist deformation appears;

2) If $E>E_{\text {th }}=f(H)$, also a splay-bend deformation is superimposed to the preceding one.

Notice that $E_{\mathrm{th}}=E_{\mathrm{c}}$ if $H \leqq H_{\mathrm{c}}$.

The relation $E_{\mathrm{th}}=f(H)$ has first been studied by Deuling $[4,5]$ in the strong anchoring hypothesis and for any elastic anisotropy; he found that in the general case it is only possible to give a numerical solution for the above mentioned problem.

In the present paper we will show a way to solve the analytical problem in a particular region, obtaining an explicit relation for the threshold electric field vs. the applied magnetic field, if the latter one is not far to its critical value $H_{\mathrm{c}}$.

\section{One constant approximation}

We represent the nematic director in the form

$$
\boldsymbol{n}(\boldsymbol{r})=(\boldsymbol{i} \cos \varphi+\boldsymbol{j} \sin \varphi) \cos \vartheta+\boldsymbol{x} \sin \vartheta,
$$

Reprint requests to G. Barbero, Dipartimento di Fisica, Politecnico, C. so Duca degli Abruzzi, 24-I-10129 Torino (Italia).

* Work supported by Centro Ricerche Fiat S.p.A.

${ }^{I}$ also of Laboratoire de Physique des Solides, Université de Paris-Sud, Orsay, France, and Dipartimento di Fisica, Università di Calabria, Arcavata di Rende, Italia.

2 also of Facoltà di Medicina Veterinaria, Università, Torino, Italia. and restrict ourselves to a problem homogeneous in the $[x, y]$-plane, i.e. $\boldsymbol{n}(\boldsymbol{r})$ is assumed to depend only on the $z$-coordinate: $\theta=\theta(z)$ and $\varphi=\varphi(z)$ are the co-azimut and the anomaly of the director, respectively (see Fig. $1 \mathrm{a}-\mathrm{b}-\mathrm{c}$ ).

In the present case Frank's formula for the free energy density of the NLC cell is given by

$$
\begin{aligned}
F(\varphi, \vartheta)=(K / 2)(\pi / d)^{2} & \left\{\dot{\vartheta}^{2}+\dot{\varphi}^{2} \cos ^{2} \vartheta-h^{2} \cos ^{2} \vartheta\right. \\
& \left.\cdot \sin ^{2} \varphi-e^{2} \sin ^{2} \vartheta\right\},
\end{aligned}
$$

where $d$ is the sample thickness, the walls coordinates being $z= \pm d / 2 ; k$ is the elastic constant; the dot represents the derivation with respect to $u \equiv \pi z / d ; h \equiv H / H_{\mathrm{c}}$ is the reduced magnetic field; $e \equiv E / E_{c}$ is the reduced electric field.

Obviously in the one constant hypothesis

$H_{\mathrm{c}}=(\pi / d) \sqrt{K / \chi_{\mathrm{a}}}$ and $E_{\mathrm{c}}=(2 \pi / d) \sqrt{\pi K / \varepsilon_{\mathrm{a}}}$,

$\chi_{\mathrm{a}}$ and $\varepsilon_{\mathrm{a}}$ being the susceptivity and permittivity anisotropy, respectively [4].

The Euler-Lagrange equations following from the position (1) are obtained by the system

$$
\left\{\begin{array}{l}
\dot{\varphi}^{2}=h^{2}\left(\sin ^{2} \varphi_{\mathrm{MAX}}-\sin ^{2} \varphi\right), \\
\ddot{y}=\vartheta\left[h^{2}\left(2 \sin ^{2} \varphi-\sin ^{2} \varphi_{\mathrm{MAX}}\right)-e^{2}\right]
\end{array}\right.
$$

near to the electric field threshold. We point out that $\varphi_{\text {MAX }}=\varphi(0)$.

Moreover, if $h \gtrsim 1$, i.e. if the magnetic field is not far from its threshold, $\varphi=\varphi_{\mathrm{MAX}} \cos u$ is deduced.

Consequently the second equation of the system (2) becomes

$$
\ddot{7}+\vartheta\left[e^{2}-\left(h \varphi_{\mathrm{MAX}}\right)^{2} \cos 2 u\right]=0,
$$

which is a Sturm-Liouville equation [6].

In order to determine the threshold value of $e^{2}$, we look for a solution of (3) of the kind

$$
\vartheta\left(u ; h \varphi_{\mathrm{MAX}}\right)=\sum_{n=0}^{\infty} \vartheta_{n}(u)\left(h \varphi_{\mathrm{MAX}}\right)^{2 n},
$$




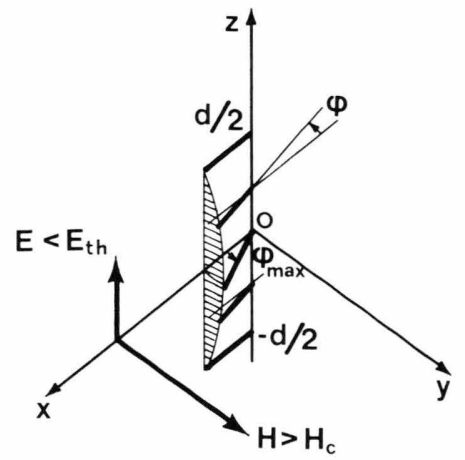

Fig. 1 a. Twist arising in the planar cell due to $H>H_{\mathrm{c}}$, $E<E_{\text {th }}$.

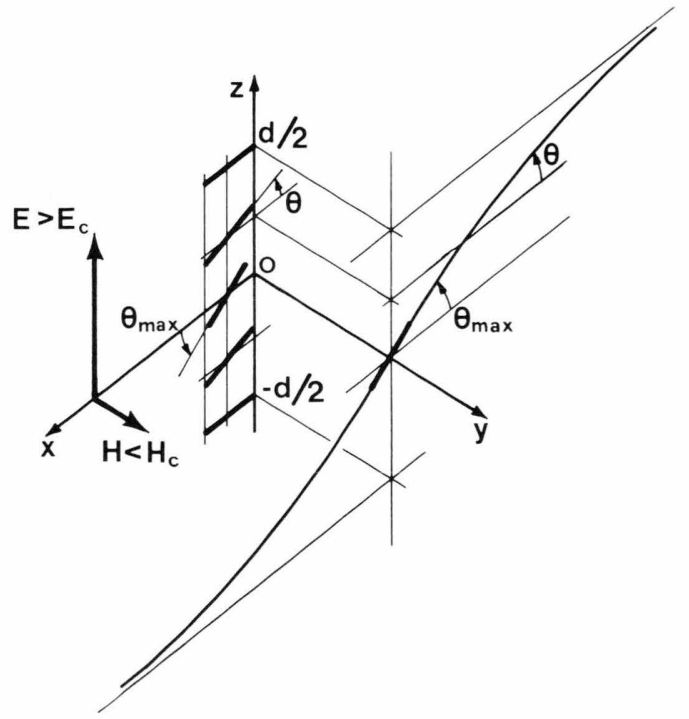

Fig. 1b. Splay-bend arising in the planar cell due to $H<H_{\mathrm{c}}, E>E_{\mathrm{c}}$.

which is surely possible, due to the Poincaré's theorem [7]. This solving method is similar to the perturbative one [8]. The symmetry of the sample with respect to the middle plane gives $\vartheta(u)=\vartheta(-u)$, which implies $\vartheta_{n}(u)=\vartheta_{n}(-u)$, and for the strong anchoring conditions $\vartheta( \pm \pi / 2)=0$, i.e. $\vartheta_{n}( \pm \pi / 2)=0$. Furthermore we put

$$
e^{2}=\sum_{n=0}^{\infty} e_{n}^{2}\left(h \varphi_{\mathrm{MAX}}\right)^{2 n} .
$$

By substituting (4) and (5) in (3) we deduce the following hierarchy of differential equations:

$$
\dot{\vartheta}_{n}+\sum_{m=0}^{n} e_{m}^{2} \vartheta_{n-m}-\vartheta_{n-1} \cos 2 u=0
$$

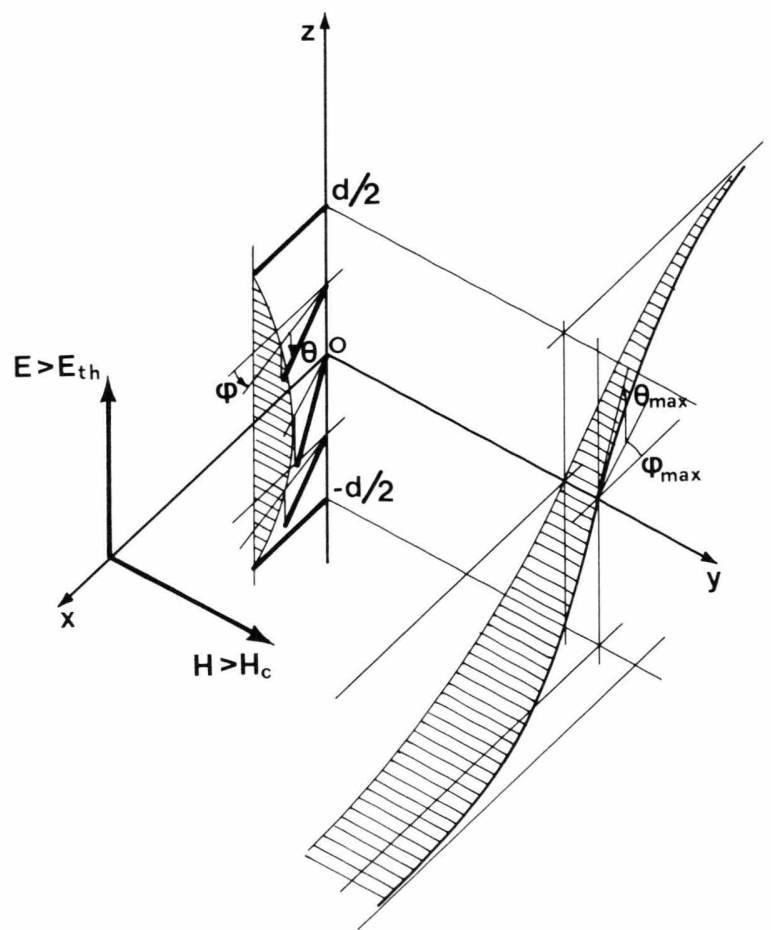

Fig. 1 c. Mixed distortion arising in the planar cell due to $H>H_{\mathrm{c}}, E>E_{\mathrm{th}}$.

for $n=0,1,2, \ldots$, and $\vartheta_{-1} \equiv 0$. Equations (6) solve the proposed problem.

In fact from (6), for $n=0$, we deduce: $\ddot{\vartheta}_{0}+e_{0}^{2} \vartheta_{0}=0$. Hence

$$
\vartheta_{0}=\vartheta_{0 \mathrm{MAX}} \cos \left(e_{0} u\right)
$$

The boundary conditions give $e_{0}=1$, that is the well known threshold, when only the electric field is applied.

For $n=1,(6)$ gives

$$
\ddot{\vartheta}_{1}+\vartheta_{1}=\vartheta_{0}\left(\cos 2 u-e_{1}^{2}\right) \text {. }
$$

By taking (7) into account it is possible to rewrite (8) as

$\ddot{\vartheta}_{1}+\vartheta_{1}=\frac{1}{2} \vartheta_{0 \operatorname{MAx}}\left[\cos 3 u+\left(1-2 e_{1}^{2}\right) \cos u\right]$.

The general solution of the ordinary linear differential equation (9) is of the kind

$$
\vartheta_{1}=\alpha \cos u+\beta \sin u+f_{1}(u)+f_{2}(u),
$$

where $\alpha$ and $\beta$ are two constants and $f_{1}(u)$ and $f_{2}(u)$ are two particular solutions of (9). We take as functions $f_{\mathrm{i}}(i=1,2)$ the solutions of the differential 
equations

$$
\ddot{f}_{1}+f_{1}=\frac{1}{2} \vartheta_{0 \mathrm{MAX}} \cos 3 u
$$

and

$$
\ddot{f}_{2}=f_{2}=\frac{1}{2} \gamma_{0 \operatorname{MAX}}\left(1-2 e_{1}^{2}\right) \cos u \text {. }
$$

It is very easy to show that a solution of (11) is

$$
f_{1}=-\frac{1}{8} \vartheta_{0 \mathrm{MAX}} \cos 3 u,
$$

while (12) yields

$$
f_{2}=\frac{1}{4} \vartheta_{0 \operatorname{MAX}}\left(1-2 e_{1}^{2}\right) u \sin u .
$$

On the other hand, symmetry considerations give $\beta=0$; moreover, a simple analysis shows that $\alpha=0$ also [9]. Then it follows that the relation (10), by substituting (11) and (12), becomes

$z_{1}=\frac{1}{4} \gamma_{0 \operatorname{MAx}}\left[-\frac{1}{2} \cos 3 u+\left(1-2 e_{1}^{2}\right) u \sin u\right]$.

By taking the boundary conditions $\vartheta_{1}( \pm \pi / 2)=0$ into account, we deduce $f_{2}=0$, and then $e_{1}^{2}=1 / 2$. Consequently

$$
\vartheta_{1}=-\frac{1}{8} \vartheta_{0 \operatorname{MAx}} \cos 3 u \text {. }
$$

By operating in the same way, routine calculation gives $e_{2}^{2}=-1 / 32$ and

$$
\vartheta_{2}=-\frac{1}{64} \vartheta_{0 \operatorname{MAX}}\left[\cos 3 u-\frac{1}{3} \cos 5 u\right] \text {. }
$$

By substituting the previous results into (5), the electric field threshold is obtained as

$$
\begin{aligned}
e_{\mathrm{th}}^{2}=1 & +\frac{1}{2}\left(h \varphi_{\mathrm{MAX}}\right)^{2}-\frac{1}{32}\left(h \varphi_{\mathrm{MAX}}\right)^{4} \\
& +O\left[\left(h \varphi_{\mathrm{MAX}}\right)^{6}\right] .
\end{aligned}
$$

By using a Landau-development for the free energy, in the case $h \geq 1$, it is easy to show that $\varphi_{\text {MAX }}^{2}=4$ $(h-1)$, as well known [10]. Hence the relation between the electric field threshold and the applied magnetic field is given by

$$
e_{\mathrm{th}}^{2} \cong 1+2 \varkappa(1-\varkappa / 4),
$$

$\varkappa \equiv h^{2} \quad(h-1)$ being the normalized reduced magnetic field. Equation (19) is a very simple analytical description of the function $E_{\mathrm{th}}=f(H)$ near the magnetic critical value $H_{\mathrm{c}}$. Such an equation is valid for $1<h \leqslant 1.2$, i.e. for $250 \mathrm{G}<H$ $\lesssim 300 \mathrm{G}$ of [4], where the considered NLC is p-nhexyloxybenzilidene-p-aminobenzilonitrile (HBAB) at $75^{\circ} \mathrm{C}$. But (19) is not applicable to the experiment of Ref. [4], since the one constant approximation is not realistic for $\mathrm{HBAB}$, presenting $K_{22}=$
$0.415 K_{11}$ and $K_{33}=2.49 K_{11}$ [4]. Nevertheless, it is possible to show that a relation of the kind (19) is valid also if the splay, twist and bend elastic constant are different from each other, i.e. if

$$
K_{11} \neq K_{22} \neq K_{33}
$$

\section{Elastic anisotropy}

In the general case of elastic anisotropy the system (2) becomes, near the electric threshold,

$$
\begin{aligned}
\dot{\varphi}^{2} & =h^{2}\left(\sin ^{2} \varphi_{\text {MAX }}-\sin ^{2} \varphi\right), \\
\ddot{q}= & =\left\{\beta h ^ { 2 } \left[(2-\alpha) \sin ^{2} \varphi\right.\right. \\
& \left.\left.-(1-\alpha) \sin ^{2} \varphi_{\text {MAX }}\right]-e^{2}\right\},
\end{aligned}
$$

where $\alpha$ and $\beta$ are Deuling's parameters [3] related to the elastic anisotropy, i.e. $\alpha \equiv\left(K_{33} / K_{22}\right)-1$ and $\beta \equiv K_{22} / K_{11}$.

Obviously $H_{\mathrm{c}}$ and $E_{\mathrm{c}}$ are in the present case defined as

$$
\begin{aligned}
& H_{\mathrm{c}}=(\pi / d) \sqrt{K_{22} / \chi_{\mathrm{a}}} \text { and } \\
& E_{\mathrm{c}}=(2 \pi / d) \sqrt{\pi K_{11} / \varepsilon_{\mathrm{a}}} .
\end{aligned}
$$

With the aim to determine the threshold electric field $E_{\text {th }}$ as a function of the crossed magnetic field $H$ in the region $H \gtrsim H_{\mathrm{c}}$, we put

$$
\begin{aligned}
& e^{* 2} \equiv e^{2}-\frac{1}{2} \alpha \beta\left(h \varphi_{\mathrm{MAX}}\right)^{2}, \\
& h^{* 2} \equiv \frac{1}{2}(2-\alpha) \beta h^{2},
\end{aligned}
$$

obtaining the second equation of the system (20) written formally as well as (3).

By applying the previous method, the threshold electric field is given, after some rearrangements, as

$$
\begin{aligned}
e_{\mathrm{th}}^{2}= & 1+\frac{1}{4}(2+\alpha) \beta\left(h \varphi_{\mathrm{MAX}}\right)^{2} \\
& -\frac{1}{128}(2-\alpha) \beta^{2}\left(h \varphi_{\mathrm{MAX}}\right)^{4}+O\left[\left(h \varphi_{\mathrm{MAX}}\right)^{6}\right] .
\end{aligned}
$$

Since $\varphi_{\text {MAX }}^{2}=4(h-1)$, we get finally

$e_{\text {th }}^{2} \cong 1+(2+\alpha) \beta \varkappa\left\{1-\chi(2-\alpha)^{2} \beta /[8(2+\alpha)]\right\}$

Such a relation generalizes (19) to the case of elastic anisotropy: obviously (19) may be deduced as a particular subset of (23), if $\alpha=0$ and $\beta=1$.

By differentiating (23) with respect to $h$, we find

$$
\left(\mathrm{d} e_{\mathrm{th}} / \mathrm{d} h\right)_{h=1}=(2+\alpha) \beta / 2 \text {. }
$$

This means that the slope of the curve $e_{\mathrm{th}} \mathrm{vs} . h$ near the critical value of the magnetic field is neither zero nor infinite (1.453 for the HBAB, 1 for an 


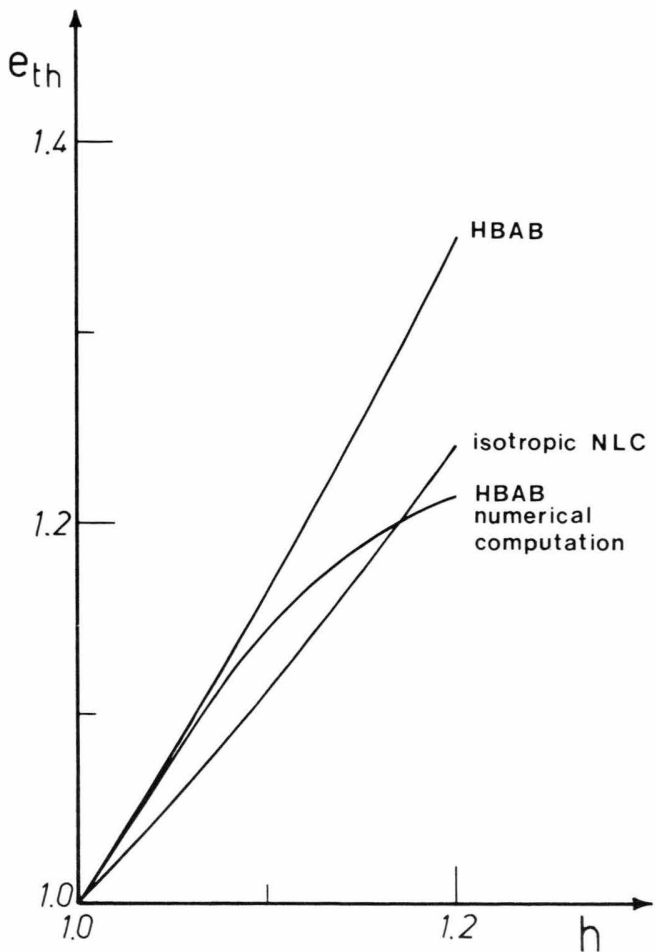

Fig. 2. Reduced electric field at the threshold vs. reduced magnetic field: analytical approximation for $\mathrm{HBAB}$ and isotropic NLC and numerical computation for HBAB.

[1] H. J. Deuling, M. Gabay, E. Guyon, and P. Pieranski, J. de Physique, 36, 689 (1975).

[2] H. J. Deuling, Mol. Cryst. Liq. Cryst. 19, 123 (1972).

[3] H. J. Deuling, in: L. Liebert "Liquid Crystals" Solid State Physics S. 14, Academic Press, New York 1978.

[4] H. J. Deuling, E. Guyon, and P. Pieranski, Solid State Comm. 15, 277 (1974).

[5] H. J. Deuling, A. Buka, and I. Jànossy, J. de Physique, 37, 965 (1976).

[6] F. G. Tricomi, Istituzioni di Analisi Superiore, CEDAM, Padova 1970, p. 271. elastically isotropic NLC), defining a second order transition.

Furthermore, since if $h \gg 1$ (i.e. $h>1.6$ )

$$
e_{\text {th }}^{2}=1+\beta h^{2}
$$

may be obtained [4], (24) and (25) could be used in order to determine experimentally the elastic ratios $K_{22} / K_{11}$ and $K_{33} / K_{11}$ from the slope of the function $e_{\text {th }}(h)$ at the critical value and from the slope of the asymptotic curve $e_{\mathrm{th}}^{2}\left(h^{2}\right)$. In conclusion, an explicit relation giving the behaviour of the electric field threshold as a function of the crossed magnetic field near to the critical value has been derived. In Fig. 2 the explicit functions $e_{\text {th }}(h)$ for the HBAB and for an isotropic NLC are shown and compared with the curve obtained by numerical computation. The analytical approximation gives an overestimate of $2 \%$ for $h=1.10$, while at $h=1.20$ the overestimate goes up to $10 \%$.

\section{Acknowledgements}

We are very grateful to L. Macera for his precious help in drawing the figures.

[7] L. Elsgolts, Differential Equations and the Calculus of Variations, MIR Moscow 1977, p. 60.

[8] G. Barbero and A. Strigazzi, Mol. Cryst. Liq. Cryst. Lett. 82, 5 (1982).

[9] L. Gatteschi, Funzioni speciali, UTET Torino 1975.

[10] E. B. Priestly, P. J. Wojtowicz, and Ping Sheng, Introduction to Liquid Crystals, Plenum Press, New York 1974, p. 115. 